\title{
REMARKS
}

oN

\section{MALIGNAN'T DISEASES}

\section{or \\ THE SKIN OF THE FACE.}

BY CASAR HAWKINS, SURGEON TO ST. GEORGE'S HOSPITAL.

READ MAY 9TH, 1837.

ThE chief object of the following remarks is to describe a peculiar form of malignant disease of the face, which does not appear to have received any distinct notice by surgical writers, although its character is so well marked, as to require a separate consideration. To make myself understood, however, I must, in the first instance, endeavour to describe briefly the more familiar forms of malignant disease, in order to compare the several varieties with each other.

But as the term malignant is employed in a very vague and ill defined manner, it is necessary to premise, that it is my intention, in these observations, to restrict it to such diseases, as essentially possess a new structure, capable of exciting a poisonous influence in one or more of these several modes; 1st, upon the neighbouring textures, which are converted into a substance, either exactly similar, or at least analogous, to that of the new formation; 2dly, upon 
the absorbent system, so that the hearest glands become enlarged into a tumour like that originally deposited; or 3dly, upon the whole constitution, so that the poisonous secretions of the newly formed part gain access to the circulating fluids, and tubercles of various forms, but of the same or analogous character, become developed in some distant organs or textures, which have no direct communication, except through the blood, with the parts in which the new structure was first formed.

-This poisonous influence is equally shewn in its several degrees, whether the malignant structure be supposed to be entirely local in the first instance, or to be preceded invariably by a peculiar predisposing condition of the whole system ; since, even on the latter hypothesis, the secretions of the local tumour still further contaminate the blood, as soon as absorption begins to take place.

By this restriction of the term, we exclude from among the malignant diseases of the face, 1st, the irritable and intractable ulcers of this part, so well described by Mr. Earle, in the 12th Volume of the Transactions of the Society; 2dly, the various forms of Scrofulous Phagedenic Ulcer, or Scrofulous Lupus, which attack the nose, eyelids and cheeks; 3dly, the several varieties of Tubercular Sebaceous disease, Tubercular Lupus, Cancer perforans, Noli me tangere, or whatever other name is adopted to designate these destructive ulcerations, which occur in the same parts; and, 4thly, the Hypertrophy of the Skin of 
the nose, described by Mr. Hey, Civadier, and other writers, and often called Cancerous Tumours, Loupes, Lipoma, and so on, though they have nothing in common with those affections. None of these are malignant in this confined sense, however large may be the tumours of the last named disease, or however extensive and destructive the ulcerations of the three preceding, because the interior of the tumours, and the hard edges, and fungous granulations of the ulcers, contain no new structure, but are a development of the natural textures, with the deposits of inflammation only, incapable of affecting other parts of the body, even when fatal to the lives of the wretched objects, who are victims to these frightful disorders.

I. The class of fungoid malignant diseases, whether in their hamatoid, medullary, or melanoid varieties, require little notice, since they seldom occur in the skin of the face, except when the constitutional taint has already evinced itself by the formation of a tumour in soine other part of the body. The forms most frequently developed in such cases are the melanotic tubercles, or a soft lardaceous kind of medullary tumour; these are seldom single, like the several forms of cancer, but are in great numbers over the face and scalp, and are too rapidly formed, towards the end of the patient's life, when he has nearly sunk under the same disease in some other part, to occasion trouble in determining their nature. For the same reason, there is seldom time for the processes of softening and ulceration, and fungoid growths; but 
if these phenomena do take place, they present nothing striking to our observation, beyond what is usually to be seen in this disorder elsewhere.

II. The class of scirrhous, or cancerous complaints, on the contrary, are very peculiar when met with in the face, and differ in many respects from what is usually called cancer, whence has arisen much difference of opinion as to their nature, and whence, consequently, have taken place some errors of practice, injurious to the reputation of the surgeon, and to the well being of the patient.

Cancer of the skin of the face is presented to our notice in three different forms, of which the most frequent may be called,

1. The common cancer of the face, with which, as it shews itself in the lower lip, most surgeons are familiar.

It commences for the most part in the form of a little hard tubercle in the substance of the cutis and subjacent cellular texture, with the little ulcer or fissure which takes place in it, covered from time to time with a thin scab. A tumour of this kind I removed from the lip of a woman 45 years of age, several years ago, since which time I have had the opportunity of knowing that no return of the disease has taken place.

To this stage succeeds a deep excavated ulcer, with a foul and painful surface, - or a mass of exuberant granulations, flabby and bleeding slightly. The original tubercle now no longer exists, or is mixed with hardened cellular substance in the neigh- 
bouring textures, and some minute scirrhous tubercles are sometimes visible in the muscles around the ulcer. Next perhaps succeeds a further stage, the ulceration having spread extensively on the inside of both lips, and having affected the gums and lower jaw, part of this bone being softened and ulcerated, and part exfoliating slightly.

The unfortunate patient is generally carried off by the disease in this stage of contamination, worn out by the irritation of the foul, foetid, bleeding and sloughing ulcers of the face and glands, or half starved or suffocated from their pressure, the tumour forming sometimes a great mass of cancerous disease, from the jaw to the sternum, with deep excavated ulcers of several inches' diameter. In a few cases, however, the poison is absorbed, and the whole system contaminated, so that tubercles of scirrhus or fungus hæmatodes are found in other parts of the body. It is enough to refer to one such case: a patient who died in St. Bartholomew's Hospital in 1833, with cancer of the lip, in whom a few scirrhous tubercles were discovered in the liver, and an immense number in every part of the heart*.

When common cancer occurs in other parts of the face, it presents exactly the same characters: viz., the excavated ulcer, with its hard everted margins and fungous growth, together with the peculiar sallowness of the countenance so expressive of malignant disease. 
The experience of every surgeon demonstrates, that although the tumour, or the ulcer which succeeds it, may often be removed with success, yet that a return is frequently to be expected, much more frequently, for instance, than in the cancer scroti; and further, that no measure can safely be trusted, except complete excision, sometimes by the hare-lip operation, at other times by the removal of a semilunar portion of the edge of the lip, according to the situation, shape and size of the disease, or occasionally by the Taliacotian method; -and in the rest of the face, by some kind of operation, adapted to the form and local circumstances of the part affected.

2. The second form of cancer of the face is one which I have been accustomed for some years to describe under the name of the ".cancerous ulcer," or "phagedenic ulcer of the face of old persons."-Its usual origin, I believe to be a flat brownish tubercle, generally situated in the angle between the cheek and the ala nasi, or in the inner canthus of the eye, which is frequently stationary for a long time before some accidental violence induces ulceration;-this tubercle is softer, flatter and darker than that of common cancer, as if it implicated the outer texture only of the cutis, including the coloured rete mucosum.

The ulcer has a dark shining appearance, with slight elevation of its edges, which are jagged and irregular, and the skin around is not thickened nor inflamed, as in ordinary cancer, from the ulcer of which it is also distinguished by the trifling pain which accompanies it,-by the absence of hæmor- 
rhage, sloughing and fungus, and by its very slow progress,-many years sometimes elapsing before very. extensive ravages have been committed by it, during which time the ulcer sometimes remains nearly stationary for a time, or becomes covered by a thin skin, in which the vessels of the subjacent texture are visible; and in these intervals of rest the new structure at the edges diminishes in thickness.

In a case of this kind a little wart (as it was called by the patient) had been ulcerated four years, at the margin of the nose, and yet had not become half an inch broad, and was only just beginning to ulcerate into the nostril. It was my intention to have cut out the diseased parts, and to have brought round a portion of the skin of the face to supply their place, when a severe and nearly fatal attack of erysipelas effected a cicatrisation of the ulcer for some time, and successive attacks of the same disease, with domestic circumstances, prevented the operation being performed before I lost sight of her, a few months ago*.

While the ulcer spreads gradually, opening into the cheek, or the malar and maxillary bones, or leaves the eyeball suspended in its socket, with destruction of the eyelids and circumference of the orbit, and partial exfoliation and softening of the bones with which the disease is connected, its difference from ordinary cancer is evinced in the most remarkable manner, by the little disturbance which it causes in

* I have seen this patient several months since this paper was read, and the ulcer had made very little more progress. 
the general health, and by the entire absence of contamination, as far as I am aware, in the absorbent glands. It possesses the same degree of power of contaminating the surrounding textures as is seen in the warty or cancerous tumours of cicatrices, which I have endeavoured to describe in the 19th volume of the Transactions, but is perhaps only malignant in this lowest degree', and is, therefore, if it does not affect the glands at all, an instance of purely local cancer, which the common cancer of the face, and the cancer scroti are often called, but very erroneously, since they affect both the glands and the general system. At all events, if it ever affect the glands, such an occurrence is very rare, and I have not seen it where the ulcer has existed a great many years, and has destroyed the patient by its local effects.

The slow progress and peculiar appearance of the ulcer have sometimes led to the disease being called lupus, or lupoid tubercle,-but I prefer the designation I have before given it of cancerous ulcer, as indicating the scirrhous nature of a new structure, possessing a malignant influence upon every texture in its neighbourhood,-yet inferior to common cancer in the degree of malignancy, since it does not contaminate the absorbent glands,-it is, in fact, what is sometimes called semi-malignant.

I met not long since with an excellent account of the ulcer of this disease, by Dr. Jacob, in the 4th volume of the Dublin Hospital Reports, under the title of an "Ulcer of a Peculiar Character, which attacks the eyelids and other parts of the face," but 
this gentleman does not seem to be aware that the origin of the disease is usually tubercular, although its malignant influence may be generally observed during its whole progress, - some new structure at the margin, or a little thin layer of a brownish white colour below the ulcer, shewing the real character of the disease.

When the ulcer spreads from the eyelid to the conjunctiva of the globe of the eye, the cancerous deposit can even then be dissected and exposed, the existence of any portion of which, after an operation, will cause the reappearance of the disease. On the other hand, the cancerous ulcer is very often confounded in description with ordinary cancer; for example, in the excellent observations on Malignant Diseases, by Mr. Travers, in the fifteenth volume of the Transactions, in addition to a graphic account of, "Cancer of the Lip," and in another section, of " Cancer of the Eyelids and contents of the Orbit," both of which are of the ordinary form of cancer, there is a third section, on "Cancer of the Face," which appears to me to include partly the "cancerous ulcer," and partly the characters of ordinary cancer : the smooth, shining surface, and slow progress of the one, with the fetid discharge, exuberant fungous granulations, and frequent hæmorrhage of the other. The best account of the whole disease, however, with which I am acquainted, is by Mr. Mackenzie, in his admirable work on the Diseases of the Eye, but under the name, without any qualification, of "Cancer of the Eyelids." 
While, however, I prefer the modified term which I have long employed of " Phagedenic or Cancerous ulcer," to designate the lower degree of malignancy of this form, in comparison with that of ordinary cancer of the face, it must be remembered that it is very difficult to destroy all the new structure of even this local disease by caustics, and also, that wherever vain and injudicious measures are adopted to heal, what in fact must be removed, the disease may be much aggravated, and made more like ordinary cancer in its progress. On the whole, it appears to me that the removal, by the knife, of the tumour or of the ulcer, is in general the safest method, but in a broad flat ulcer, without any depth of new structure, I prefer the employment of the chloride of zinc, as lately introduced into practice in this country by Mr. Ure, after witnessing its use by Dr. Cangurin, which I have frequently had recourse to without any of the injurious effects of other caustics.

3. The third form of malignant disease of the scirrhous kind in the skin of the face, may be called the Cancerous Tumour or Fungous Cancer of the Face of old persons, of which I am not aware that any account has been written.

The early stage of this disease is presented to us in the appearance of a small round or oval tumour in the skin, generally in the cheek, or over the malar bone, or on the ala nasi. It is nearly of the natural colour of the skin for a long while, or is a little whiter, from the outer part of the cutis being thinned by the growth of the tumour, so as to allow the 
colour of its interior to shine through it. A section of the tumour shews it to be white, solid, but not very firm, lardaceous in consistence, rather than of the firm hardness of ordinary cancer. It has a well defined margin, separate from the rest of the skin, and where it projects below the cutis it is covered by a kind of cyst.

The tumour is more globular, soft, insulated and distinct, more completely confined to the texture of the skin, more elevated and less liable to become puckered, than ordinary cancer of the skin of the face, and less liable to have lancinating pain before the ulcerative stage has begun.

It is more elevated and circular, and of a whiter colour, more abrupt at its margin, and extends deeper into the substance of the cutis than the tubercle of the cancerous ulcer.

It has fewer vessels ramifying on its surface, and has none of the livid colour, previous to its ulceration, of fungus hæmatodes, nor of the darkness of structure of melanosis, and its texture is firmer and more organized than that of medullary tumours. It is distinguished too from all these diseases, by its being single, and by the length of time that it remains stationary.

If it forms upon the nose, it is easily distinguished from the tumours of hypertrophy of the ala nasi, by the absence of surrounding redness and thickening, by its defined cyst-like limits, and by its having none of the enlarged sebaceous follicles observed in that disease. 
The tumour grows thus smooth, globular, and nearly unattended with pain, to the size of a nut, or of a walnut, before it excites apprehension in the patient's mind. At last it is pricked or irritated, or ulcerates spontaneously, and there arises a mass of healthy granulations from the surface, which spread out considerably beyond the tumour, over the surrounding skin, to the height of an inch or more, with a copious dis:charge of healthy pus, without foetor, and without sloughing or bleeding, and not even now very painful. The tumour at the basis of these granulations increases in depth and in diameter, but is free for a long time from any attachment by altered cellular texture to the subjacent parts, so as still to allow of removal with every chance of success. This stage is seen in drawings* 4 and 5 , and in the preparations of the ulcerated tumours subsequently removed from the same patient, which, however, it may be observed, (like all vascular tumours) are not half so large as they were before their removal. The disease returned in one of these cases (for reasons which will hereafter be mentioned); in the larger of the two the disease had not returned for two or three years after the operation, when I saw the patient last.

A comparison of these drawings with the former shews how different the circular prominent fungus of this disease appears from the soft irregularly formed granulations, and the excavated ulcer with everted

* These and other drawings, together with preparations and casts illustrative of the descriptions in the paper, were exhibited.-ED. 
margins of the common cancer; and from the flat, dark coloured surface, destitute of granulations of the phagedenic ulcer; neither has it any resemblance to the bleeding, sloughing surface of fungus homatodes.

The tumour grows to a considerable size before it alters its character, and before the general health suffers much, as will be evident by an examination of a cast on the Table, in which the prominent mass occupies the whole side of the nose, and formed an ulcerated tumour, three inches in diameter, and nearly two in thickness. The appearance of the surface was here a little altered before the cast was taken, and its prominence lessened by the application of a solution of arsenic, with some diminution of the pain the patient experienced from the disease, which had commenced fourteen months previously; part of the tumour had been then removed by a ligature by a very distinguished surgeon, no doubt from his not being aware of its true nature. The form of cancerous tumour in this patient, although of so large a size, is well contrasted with common cancer of the same part, in the two casts; both patients died about four months after they were taken, and yet the subject of the former had none of the peculiar leaden hue in the countenance illustrative of the more malig. nant variety in the latter case.

After a time ulceration extends more deeply into the tumour, and its projecting appearance is lost; the bones and deeper parts become rapidly changed into the new structure, which, in some parts, is gristly 
like scirrhus, but in others is softer and more pulpy, like some cases of medullary disease of the bones. The ulcer in this stage is also somewhat intermediate in eharacter between these two diseases.

R. B., æt. '59, was admitted into St. George's Hospital in April 1835, the disease having commenced about twelve months previously by a small lump on the bridge of the nose, which ulcerated in six months, when of the size of a sixpence, attended subsequently with a good deal of pain. There was on his admission a rather foul ulcer an inch in diameter, on the surface of a fungous mass, which $I$ was induced to believe, from its appearance, was an instance of this peculiar fungous cancer. The tumour reached transversely from one maxillary bone to the other, and from the frontal spine nearly to the tip of the nose, - part of the fungous granulations projecting into the nostril, and the nasal bones having been converted into a soft mass with some loose spiculæ of bone. I at first thought of attempting the removal of the tumour, and bringing down some of the skin of the forehead to form a new nose, but it soon became evident that the disease had begun to affect the frontal bone, and was attached firmly to the maxillary bones at the side of the nose; - the poor man was also weak and thin, and had been totally blind from amaurosis for some years, and seemed therefore unfit for such an operation. The tumour gradually spread laterally, and ulcerated more extensively, and in November following presented the appearance exhibited in the drawing. He 
died in February 1836, about two years after the disease commenced.

The brain was healthy, with the exception of a small quantity of water. The optic nerves passed close to the disease, the bone through which they passed just beginning to be affected by the disease; they were considerably flattened, and darker coloured than natural, but had their healthy rotundity and appearance near the eyes. The amaurosis being long antecedent to the appearance of the tumour of the skin, had of course no connexion with it. The parts affected by the disease can be seen in a preparation. The frontal bone was just beginning to be softened and rough by the disease where the nasal bones had originally joined it, all trace of which was lost. The dura mater lining the interior of the æthmoid bone, and part of the sphænoid, and of the frontal around the'æxthmoid, shewed a few small projections of the disease into the cranial cavity; a great part of these bones, together with the nasal parts of the maxillary and palatine bones, having been converted into a whitish tumour, in consistence between scirrhus and fungus medullaris, all the cells of the nose being filled with a similar substance; where subjected to more pressure however, and therefore probably growing less rapidly, it resembled scirrhus. The antrum on each side was clear and healthy. The soft palate, in contact with the palatine plates of the palate and maxillary bones, was thick and hard, as if scirrhous. The sphænoid cell contained a little thick pus, and a part of the bone was dead, and 


\section{MR. HAWKINS ON MALIGNANT DISEASES}

partly loose. The olfactory nerves going through the tumour preserved their natural appearance. The liver was granulated, but all the other viscera were healthy, and no gland appeared to have been contaminated.

The patient from whom the fourth drawing was taken was a man, aged 79, who was admitted into St. George's Hospital in July 1833, with the small tumour on the cheek there represented, which had begun eight months before, and had been ulcerated three months. The tumour was removed, but a small tumour reappeared under the cicatrix, no doubt from a very fine portion of the tumour, which I discovered running perpendicularly towards the malar bone, by the side of the chief part of the tumour, which had been cut across and unfortunately escaped observation. I wished to have again removed the tumour, with the malar bone, to which it was attached, but he would not submit to it. In January following, an ulcer formed in the new tumour, with much pain, and a small gland was felt hardened under the chin, and two others at the margin of the sterno mastoid muscle. He continued to linger till November 1834, about two years from the beginning of the disease.

Before his death the tumour extended considerably over the temple, and over the upper and lower jaws, and nearly to the nose, there being in the centre a large deep ulcer, the malar and maxillary bones having been destroyed, so that the probe passed down to the bottom of the orbit, full four inches 
deep, and might, in fact, have been made to enter the brain. The surface of the ulcer to the last, was generally healthy, red, and granulating, and nearly free from bleeding and from sanious or fætid discharge, though it had much of the severe lancinating pain of scirrhus.

The dissection was very interesting. One preparation shows the size of the ulcer, and the scirrhous nature of the tumour, which remained at the time of his death, a section having been made of one portion, which is turned back for this purpose;-it shows also the formation of this new substance over the surface of the eyeball and its muscles, where they have been left insulated by the ulceration, the tendon of the temporal muscle, and the coronoid process of the lower jaw having been also connected with the same structure.-The malar bone, the zygomatic process, and the front of the antrum were destroyed, its membrane being unaltered, and the duct of the parotid gland was destroyed, the flow of saliva from it having been very distressing to the patient during his life. The same preparation shows also the manner in which the temporal parts of the sphænoid and temporal bones were concocted into new substance, softened and ulcerated by two openings, one of them half an inch in diameter, which led into the interior of the cranium;-to these openings the brain adhered, the dura mater having also ulcerated; and the hemisphere of the cerebrum, which was much flattened when first exposed, presented a large cavity capable of containing several ounces of fluid, extend- 
ing through the whole length of the middle and posterior lobes of the cerebrum. This cavity contained no pus, which had doubtless escaped through the opening at the bottom of the orbit, but the interior was vascular, and the brain was darker and yellowish, as round an apoplectic effusion. It was remarkable that, notwithstanding this extensive disease, the mind of the patient had not been in the least affected during his life.

Two or three glands were enlarged and hardened; -one had within it some cheesy matter, and another some green pus, the structure of the substance of the glands being probably scirrhous, though not very distinctly so.

One kidney contained an aqueous encysted tumour in its substance, the size of a nut, and had another, holding six or eight ounces of water, attached to its outside, and making the surface of the organ concave from its pressure. These cysts were of the nature of those described by me in the 18th volume of the Transactions, and are of course not to be considered as any evidence of malignant disease. In the lower part of the liver, however, was a circumscribed circular tumour, about four inches in diameter, of a dark colour, solid and vascular, and with some marks of effused blood in its substance; it seemed to be a deposit of new substance in the texture of the liver, without defined limits, and though it does not exactly resemble the usual forms of fungus hæmatodes in the liver, yet it can hardly be examined without being supposed to be a ma- 
lignant tumour, more like that form of disease than any other with which $I$ am acquainted.

It appears to me then that when the cancerous tumour of the face has reached its third stage of advanced ulceration, it bears more resemblance than it previously does to common cancer of the lips and face; but it is attended with more tumefaction around and beneath the ulcer,- the edges are less curled and hardened,-the discharge is healthy purulent secretion, instead of offensive watery and sanious fluid of a peculiar odour; - and there is much less disposition to blecding and sloughing.

It is easily distinguished from the Phagedenic ulcer of the largest size by its tumefaction and fungous growth, by its granulating and vascular surface, by the depth and extent to which the subjacent parts are excavated and converted into new structure, by the greater pain which accompanies it, and by the rapidity of its progress:-its final and fatal stage being attained in about two years, instead of, perhaps, twenty or thirty.

'The fungous growth, where not subjected to pressure, and growing therefore more rapidly, as in the cells of the nose, is much like what takes place in some cases of fungus hæmatodes or medullary tumour, but for the most part it is of a scirrhous character, and may be considered, perhaps, like many tumours, to be in a manner intermediate between or to partake of the nature of the two classes of cases. 'The ulceration, however, differs from that of fungus hematodes, as much as it does from that of common 
cancer, in having none of that rapid sloughing and bleeding, characteristic of tumours of that description.

In malignancy it is intermediate between the cancerous ulcer and the common cancer;-more rapidly and extensively contaminating the surrounding parts than the former, but not having the neighbouring scirrhous tubercles, and scirrhous bands of cellular texture, met with in the latter disease, and admitting, therefore, of removal by the knife, if sufficient care be taken to excise the whole, with more chance of the cicatrix remaining sound, than in ordinary cancer, -in fact, with almost a certainty of success, where it has not attained a great magnitude.

With regard to the absorbent system, the last case would seem to show that the cancerous tumour does affect it, which is never the case, as far as I know, in the cancerous ulcer of the face ; - yet the enlargement of the glands is, at all events, very rare, and we need entertain very little fear of a return of the disease in the glands, after removal of the tumour. In common cancer, on the other hand, the contamination of the glands is very common, and frequently destroys the patient after the operation, even when the cicatrix has remained sound.

Finally, it would seem, from the tumour of the liver in the last case, that the whole constitution may be impregnated with the poison of this complaint, in which respect also the cancerous tumour is more malignant than the phagedenic ulcer, in which $I$ do not know that such an occurrence has ever been ob- 
served. But in this point too it is probably surpassed by the common cancer of the face, in which form of disease scirrhous tubercles of the liver or some other organ, (it has already been remarked,) have occasionally been observed; although even in this, the most malignant form of cancerous disease of the face, the simultaneous development of the poison in other organs or textures is rare; in comparison with cases of cancer or fungus hæmatodes of most other parts of the body. Therefore, if the tumour of the fungous cancer is carefully removed by the knife, and not trifled with by caustics, and no gland is enlarged at the time of removal, the prospect of cancer becoming developed in some other part of the body, though not impossible, is too remote to excite any apprehension of a failure of the operation from this cause.

P.S. Some months after this paper was read to the Society, a work on Tumours was published by an American author, Dr. Warren, which contains a description of the cancerous ulcer, in many respects correct, under the name of Lepoides, from the barklike scaly crust formed on the surface of the little brown tumour, before it has been excited into ulceration. As however the crust from which the name is derived, is only an occasional circumstance, not often found, it does not appear to me to be a good term by which to designate the disease, especially if $I$ am correct in believing it to be a variety of cutaneous 
90 Mr. haWkins on malignant diseases, etc.

cancer. I am glad to find, however, that this gentleman, like myself, is of opinion that the malignancy of the ulcer is confined to its immediate neighbourhood.

31, Half-Moon Street,

May 2, 1837. 